\title{
A Parallel Prime Edge-Length Crystallographic FFT
}

\author{
Jaime Seguel and Daniel Burbano \\ University of Puerto Rico at Mayaguez, Mayaguez PR 00680, USA \\ (Jaime.Seguel) (dburbano) @ece.uprm.edu
}

\begin{abstract}
Although other methods are available, computational X-ray crystallography is still the most accurate way of determining the atomic structure of crystals. For large scale problems such as protein or virus structure determination, a huge amount of three-dimensional discrete Fourier transforms (DFT) conform the core computation in these methods. Despite the fact that highly efficient fast Fourier transform (FFT) implementations are available, significant improvements can be obtained by using FFT variants tailored to crystal structure calculations. These variants, or crystallographic FFTs, use a-priori knowledge of the specimen's crystal symmetries to lower the operation count and storage requirement of a usual, asymmetric FFT. The design and implementation of crystallographic FFTs brings about several problems of its own. And, as is usually the case with prime length FFTs, prime edge-length crystallographic FFTs pose the hardest challenges among them. This paper develops and tests a parallel multidimensional crystallographic FFT of prime edge-length, whose performance is significantly better than that of the usual FFT.
\end{abstract}

\section{Introduction}

Let $Z^{d} / N=Z / N \times \cdots \times Z / N$, be the Cartesian product of $d$ copies of $Z / N$, the set of integers modulo $N$. The $d$-dimensional discrete Fourier transform (DFT) of edge length $N$ of a real- or complex-valued mapping $f$ defined in $Z^{d} / N$ is the linear operator

$$
\hat{f}(\boldsymbol{k})=\frac{1}{\sqrt{N}} \sum_{\boldsymbol{l} \in Z^{d} / N} f(\boldsymbol{l}) w_{N}^{\boldsymbol{k} \cdot \boldsymbol{l}}, \quad \boldsymbol{k} \in Z^{d} / N
$$

where $w_{N}=\exp (-2 \pi i / N)$, d denotes the dot product, and $i=\sqrt{-1}$. By endowing $Z^{d} / N$ with an order (for example, the lexicographic order), (11) can be thought as the product of a $N^{d} \times N^{d}$ complex matrix by a vector. If $d=1$, we denote this matrix $F_{N}$. Thus, (1) is computed in $O\left(N^{2 d}\right)$ time. For $d=1$, a fast Fourier transform [3] (FFT) computes (11) in $O(N \log N)$ operations. For $d \geq 2$, the usual FFT method consists of applying $N^{d-1}$ one-dimensional FFTs along each of the $d$ dimensions. Thus, (1) is computed in $O\left(N^{d} \log N\right)$ time. This complexity bound cannot be improved for general DFT computations. For 
problems whose data is endowed with redundancies, such as x-ray crystal diffraction intensity data, attempts have been made to reduce the FFT run time. A starting point in these attempts is to eliminate all redundancies induced by the presence of a crystallographic symmetry both, from the input and output data sets. The resulting input and output data sets are usually called fundamental data sets. This data reduction is indeed a lossless compression, since each data set can be reconstructed from the corresponding fundamental data set by applying symmetry relations. In order to compute (11) within fundamental data sets, the DFT equations have to be modified. This modification, which consists mainly in factoring repeated input in the DFT sum (11) and eliminating redundant outputs, reduces the operation count of the DFT. However, the complexity order of computing with the reduced system of DFT equations remains within $O\left(N^{2 d}\right)$, and therefore, DFT reductions do not produce a fast Fourier transform method. The design of fast algorithms for computing the reduced DFT equations, collectively known as crystallographic FFTs, poses several mathematical and algorithmic challenges, which are summarized is Sect. 22 These challenges, most of them still open problems, are most probably the main reason behind the rather slow emergence of crystallographic FFT methods in the scientific computing community. A crystallographic FFT for composite edge-lengths was first introduced by Ten Eyck in [7]. This method is applicable to a few crystal symmetries. A universal crystallographic FFT of composite edge-length, this is a crystallographic FFT for all crystal symmetries, is proposed in [6]. In [6] it is also shown that composite edge-length crystallographic FFTs are computed in $O(M \log M)$ time, where $M$ is the size of the subset of non-redundant input data. A prime edge-length crystallographic FFT method was first introduced by Auslander et. al. in [1]. Although this method is universal, its time complexity is on average, higher than that of the usual FFT [5]. A method whose time complexity varies with the symmetry from an optimal $O(M \log M)$ to a worst case bound smaller but close to Auslander's bound, is proposed in [5]. In the same article, parallel computing is suggested as a way of speeding up the execution time of problems that yield worst case complexity bounds. In this paper we explore such alternative by designing and testing a parallel version of the prime edge-length crystallographic FFT introduced in [5]. Our preliminary computer results show the validity of this approach.

This article is organized as follows: in Sect. 2 we review the main concepts and issues related to crystallographic FFT design. Section 3 provides a detailed description of the parallel crystallographic algorithm, and Sect. 4 summarizes the results of our computer experiments.

\section{Background}

For the rest of this paper, we assume that $N$ is prime. A matrix or vector is said to be over $Z / N$ if all its entries are in $Z / N$. All operations with matrices and vectors over $Z / N$ are performed in modulo $N$. A square matrix over $Z / N$ is nonsingular, if its determinant is different from zero in modulo $N$. 
The atomic structure of a crystal can be determined from X-ray diffraction intensity data through a intensive computer procedure. Measured intensities provide only the magnitudes $|E|$ of the normalized structure factors $E=|E| \exp (i \phi)$ of the crystal specimen. The elucidation of the crystal structure requires a knowledge of the phase $\phi$, which is unobtainable from any physical experiment. The problem of reconstructing phases from magnitudes, or phase problem, is solved by evaluating numerous trial structures. Each trial structure is refined through a sequence of steps which involve three-dimensional discrete Fourier transform (DFT) calculations. For instance, one of the most successful methods, the shakeand-bake method 8], 9, alternates optimization in Fourier space with filtering in real space, performing thus, a forward and a backward three-dimensional DFT at each refinement step. Since crystal structures consist of repeating symmetric unit cells, their intensity data is highly redundant. In mathematical terms, the observed intensities are represented by a real mapping $f$ mapping defined on $Z^{d} / N$, and redundancies described by the action of $S$, a $d \times d$ nonsingular matrix over $Z / N$ satisfying

$$
f(\boldsymbol{l})=f(S \boldsymbol{l}) \quad \text { for all } \boldsymbol{l} \in Z^{d} / N .
$$

Matrix $S$ is called a symmetry and $f$ is said to be $S$-symmetric. For example, the mapping $f$ defined in $Z^{2} / 5$ by the two-dimensional data array $[f(k, l)]_{(k, l)}$

$$
f\left(Z^{2} / 5\right)=\left[\begin{array}{cccccc}
8 & 2.2 & 5.9 & 5.9 & 2.2 \\
2.2 & 4 & 1.2 & 6 & 4 \\
5.9 & 6 & 7.7 & 7.7 & 1.2 \\
5.9 & 1.2 & 7.7 & 7.7 & 6 \\
2.2 & 4 & 6 & 1.2 & 4
\end{array}\right]
$$

is $S$-symmetric with

$$
S=\left[\begin{array}{rr}
0 & 1 \\
-1 & 0
\end{array}\right]=\left[\begin{array}{ll}
0 & 1 \\
4 & 0
\end{array}\right] \text { modulo } 5 \text {. }
$$

Clearly, an $S$-symmetric mapping $f$ satisfies $f\left(S^{j} \boldsymbol{a}\right)=f(\boldsymbol{a})$ for all $j$. Thus, the restriction of $f$ to the subset of $Z^{d} / N$

$$
O_{S}(\boldsymbol{a})=\left\{S^{j} \boldsymbol{a}: j \text { integer }\right\}
$$

yields a constant function. The subset $O_{S}(\boldsymbol{a})$ is called $S$-orbit of $\boldsymbol{a}$. It follows that an $S$-symmetric function is completely determined by its values on a fundamental set $\mathcal{F}_{S}$ formed by one and only one element from each $S$-orbit. For example, the $S$-orbits and their images under $f$ for symmetry (3) are

$$
\begin{aligned}
O_{S}(0,0)=\{(0,0)\}, f\left(O_{S}(0,0)\right) & =\{8\} \\
O_{S}(0,1)=\{(0,1),(1,0),(0,4),(4,0)\}, f\left(O_{S}(0,1)\right) & =\{2.2\} \\
O_{S}(0,2)=\{(0,2),(2,0),(0,3),(3,0)\}, f\left(O_{S}(0,2)\right) & =\{5.9\} \\
O_{S}(1,1)=\{(1,1),(1,4),(4,4),(4,1)\}, f\left(O_{S}(1,1)\right) & =\{4\}
\end{aligned}
$$




$$
\begin{aligned}
& O_{S}(1,2)=\{(1,2),(2,4),(4,3),(3,1)\}, f\left(O_{S}(1,2)\right)=\{1.2\} \\
& O_{S}(1,3)=\{(1,3),(3,4),(4,2),(2,1)\}, f\left(O_{S}(1,3)\right)=\{6\} \\
& O_{S}(2,2)=\{(2,2),(2,3),(3,3),(3,2)\}, f\left(O_{S}(2,2)\right)=\{7.7\} .
\end{aligned}
$$

A fundamental indexing set for this symmetry is then,

$$
\mathcal{F}_{S}=\{(0,0),(0,1),(0,2),(1,1),(1,2),(1,3),(2,2)\}
$$

This choice of fundamental indexing set is not unique. It is easy to show that if $f$ is $S$-symmetric, then $\hat{f}$ is $S_{*}$-symmetric, where $S_{*}$ is the transpose of the inverse of $S$. Therefore, $\hat{f}$ is also determined by its values in a fundamental set $\mathcal{F}_{S_{*}}$. We call $f\left(\mathcal{F}_{S}\right)$ and $\hat{f}\left(\mathcal{F}_{S_{*}}\right)$ fundamental input data and fundamental output data, respectively. In example (3) a fundamental input data set is

$$
f\left(\mathcal{F}_{S}\right)=\{8,2.2,5.9,4,1.2,6,7.7\}
$$

while, as a straight computation shows,

$$
\hat{f}\left(\mathcal{F}_{S_{*}}\right)=\{116,-11.1602,13.6602,6.1133,-13,11,14.3867\}
$$

is a fundamental output data set. The reduction of the usual $N^{d}$-point input data set to a subset of fundamental input data induces a modification on Eq. (1). In fact, since $f\left(S^{j} \boldsymbol{a}\right)=f(\boldsymbol{a})$ for all $j$, the input datum $f(\boldsymbol{a})$ can be factored out of the terms of $\hat{f}(\boldsymbol{k})$ that are indexed in $O_{S}(\boldsymbol{a})$ in (1). This is,

$$
\sum_{\boldsymbol{l} \in O_{S}(\boldsymbol{a})} f(\boldsymbol{l}) w_{N}^{\boldsymbol{k} \cdot \boldsymbol{l}}=f(\boldsymbol{a})\left(\sum_{\boldsymbol{l} \in O_{S}(\boldsymbol{a})} w_{N}^{\boldsymbol{k} \cdot \boldsymbol{l}}\right)=f(\boldsymbol{a}) K_{N}(\boldsymbol{k}, \boldsymbol{a}) .
$$

The expression $K_{N}(\boldsymbol{k}, \boldsymbol{a})=\sum_{\boldsymbol{l} \in O_{S}(\boldsymbol{a})} w_{N}^{\boldsymbol{k} \cdot \boldsymbol{l}}$ is the so-called symmetrized DFT kernel. The linear transformation

$$
\hat{f}(\boldsymbol{k})=\sum_{\boldsymbol{a} \in \mathcal{F}_{S}} K_{N}(\boldsymbol{k}, \boldsymbol{a}) f(\boldsymbol{a}), \quad \boldsymbol{k} \in \mathcal{F}_{S_{*}},
$$

is called symmetrized DFT. It can be shown that of $\mathcal{F}_{S}$ and $\mathcal{F}_{S_{*}}$ have the same size $M$. Thus, Eq. (17) computes the symmetric DFT in $O\left(M^{2}\right)$ time. For instance, in example (3), the symmetrized DFT involves approximately $7^{2}=49$ complex multiplications, and $7 \cdot 6=42$ complex additions. This is certainly less than the approximately $25^{2}=625$ complex multiplications and $25 \cdot 24=600$ complex additions required by the usual DFT. However, since the size of the fundamental set is a constant fraction of $N^{d}$, in terms of asymptotic growth, the mere reduction of redundant data does not yield a superior FFT method. Auslander et. al. [1] propose an algorithm for computing the core of (17) with fast cyclic convolutions. Fast cyclic convolutions are $O(Q \log Q)$ methods for computing a matrix by vector product $Y=H X$, where $H$ is a $Q \times Q$ circulant. The use of fast cyclic convolutions is expected to lower the method's asymptotic 
complexity growth. However, as pointed out in [5], even for some very common crystallographic symmetries Auslander's method involves such a large number of cyclic convolutions that its complexity is often higher than the FFT complexity boundary. In what follows, we examine basic ideas underlying the use of fast cyclic convolutions in crystallographic FFT computations. As in [1] and [5], we declare a $Q \times Q$ matrix $H$ to be circulant if it satisfies $H(k, l)=H\left(k^{\prime}, l^{\prime}\right)$ whenever $k+l=k^{\prime}+l^{\prime}$ modulo $Q$. It is easy to show that if $H$ is circulant,

$$
\Delta(H)=F_{Q} H F_{Q}
$$

is a $Q \times Q$ diagonal matrix. Thus, $Y=H X$ is computed in $O(Q \log Q)$ time through

$$
Y=F_{Q}^{-1} \Delta(H) F_{Q}^{-1} X
$$

Equation (19) is often referred as fast cyclic convolution algorithm. Fast cyclic convolutions are also used for computing $Y=T X$. where $T$ is a $P \times R$ matrix that satisfies $T(k, l)=T\left(k^{\prime}, l^{\prime}\right)$ whenever $k+l=k^{\prime}+l^{\prime}$. We call such matrices, pre-Hankel. A pre-Hankel can always be embedded in a circulant $H$. This means that $T$ occupies the upper leftmost corner of $H$. In such a case, $H$ is a $Q \times Q$ with $Q \geq P+R-1$ [5]. Auslander et. al. turn the core computations in (17) into pre-Hankel blocks. Central to this aim is the fact that for each $n \neq 0$ in $Z / N$, there is an integer $0 \leq j \leq N-2$, such that $n=g^{j}$. Auslander et. al. [1] use this property for partitioning both $\mathcal{F}_{S}$ and $\mathcal{F}_{S_{*}}$ into subsets of the form

$$
O_{g}(\boldsymbol{a})=\left\{g^{j} \boldsymbol{a}: j=0, \ldots, N-2\right\}
$$

Each pair formed by one such subset in $\mathcal{F}_{S_{*}}$ and one in $\mathcal{F}_{S}$ corresponds to a preHankel block in (17). Since $O_{g}(\boldsymbol{a})$ contains at most $N-1$ elements, Auslander's crystallographic FFT involves $\Lambda=O\left((M /(N-1))^{2}\right)$ cyclic convolutions. But since $M$ is $O\left(N^{3}\right), \Lambda=O\left(N^{4}\right)$, which is higher than the usual FFT. A way around this problem is suggested in [5]. The main idea in [5] is using orbit segments generated by a $d \times d$ matrix $C$ over $Z / N$, instead of (20). In [5], a significant reduction in the number of cyclic convolutions is shown, including reductions from $\Lambda=O\left(N^{4}\right)$ in Auslander's method to $\Lambda=1$. In these cases, the proposed crystallographic FFT achieves an optimal lower time complexity of $O(M \log M)$. Unfortunately, such optimal reduction is not always achievable, since, $\Lambda$ is bounded below by a constant that depends on the crystal symmetry. In [5], a method that parallelizes most of the cyclic convolution computations, is outlined. In the next section, we review the main ideas behind this method and provide a detailed description of its parallel version, which we call prime crystallographic FFT, (PCFFT). Our experiments confirm the potential of the PCFFT method for overcoming the execution time limit imposed by the lower bound of $\Lambda$. 


\section{Parallel Prime Edge-Length Crystallographic FFT (PCFFT)}

The crystallographic FFT proposed in [5] is derived with the help of a $d \times d$ matrix over $Z / N$, which commutes with the symmetry $S$. This matrix is precomputed through an exhaustive, direct search, which is out of the scope of this article. We recall, however, that as shown in [5], the action of $C$ partitions $\mathcal{F}_{S}$ into subsets of the form

$$
\left\{C^{j} \boldsymbol{b}: 0 \leq j \leq L_{\boldsymbol{b}}-1\right\}
$$

Here, $\boldsymbol{b}$ ranges over some $I_{S} \subset \mathcal{F}_{S}$, and $L_{\boldsymbol{b}}$ is the number of elements in (21). Similarly, $\mathcal{F}_{S_{*}}$ is partitioned into subsets generated by the action of $C^{t}$, the transpose of $C$, on a subset $I_{S_{*}} \subset \mathcal{F}_{S_{*}}$. For each pair $(\boldsymbol{a}, \boldsymbol{b})$ in $I_{S_{*}} \times I_{S}$, and $k$, $l, k^{\prime}$, and $l^{\prime}$ nonnegative integers such that $k+l=k^{\prime}+l^{\prime}$,

$$
\begin{aligned}
K_{N}\left(C^{t^{k}} \boldsymbol{a},(C)^{l} \boldsymbol{b}\right) & =K_{N}\left(C^{t^{k+l}} \boldsymbol{a}, \boldsymbol{b}\right) \\
& =K_{P}\left(C^{t^{k^{\prime}+l^{\prime}}} \boldsymbol{a}, \boldsymbol{b}\right) \\
& =K_{N}\left(C^{t^{k^{\prime}}} \boldsymbol{a}, C^{l^{\prime}} \boldsymbol{b}\right)
\end{aligned}
$$

showing that the block $W_{(\boldsymbol{a}, \boldsymbol{b})}$ whose entries are $W_{(\boldsymbol{a}, \boldsymbol{b})}(k, l)=K_{N}\left(C^{k} \boldsymbol{a}, C^{t^{l}} \boldsymbol{b}\right)$, $0 \leq k<L_{\boldsymbol{a}}$, and $0 \leq j<L_{\boldsymbol{b}}$; is pre-Hankel. The core computations in (17) are then expressed as

$$
Y=\left[W_{(\boldsymbol{a}, \boldsymbol{b})}\right]_{(\boldsymbol{a}, \boldsymbol{b})}\left[f\left(\mathcal{F}_{S}\right)\right] .
$$

These computations include neither the input datum $f(\mathbf{0})$ nor the output datum $\hat{f}(\mathbf{0})$. Computations with these data conform the so-called border computations. In order to turn core computations into cyclic convolutions, each $W_{(\boldsymbol{a}, \boldsymbol{b})}$ is embedded into a circulant. Following [5], we propose a common size $Q \times Q$ for these circulant. Thus. $Q \geq 2 L-1$, where

$$
L=\max \left\{L_{\boldsymbol{a}}: \boldsymbol{a} \in I_{S_{*}}\right\}=\max \left\{L_{\boldsymbol{b}}: \boldsymbol{b} \in I_{S}\right\} .
$$

Since the choice of $Q$ is also guided by the efficiency of the $Q$-point FFT, it is reasonable to assume that $2 L-1 \leq Q \leq 2^{\left\lceil\log _{2}(2 L-1)\right\rceil}$, where $\lceil x\rceil$ is the smallest integer that is greater than or equal to $x$. Let $H_{(\boldsymbol{a}, \boldsymbol{b})}$ be the $Q \times Q$ circulant in which $W_{(\boldsymbol{a}, \boldsymbol{b})}$ is embedded. Then, the core computations are represented as $\boldsymbol{U}=H \boldsymbol{V}$ where

$$
H=\left[H_{(\boldsymbol{a}, \boldsymbol{b})}\right]_{(\boldsymbol{a}, \boldsymbol{b})}
$$

is a block matrix. The input vector $\boldsymbol{V}$ is composed of $Q$-point vector segments $\boldsymbol{V}_{\boldsymbol{b}}$, each consisting of the $L_{\boldsymbol{b}}$ values of $f\left(C^{j} \boldsymbol{b}\right), 0 \leq j<L_{\boldsymbol{b}}$; followed by $Q-L_{\boldsymbol{b}}$ zeros. Vector $\boldsymbol{U}$, in turn, is composed by $Q$-point segments denoted $\boldsymbol{U}_{\boldsymbol{a}}, \boldsymbol{a} \in I_{S_{*}}$, that result from

$$
\boldsymbol{U}_{\boldsymbol{a}}=\sum_{\boldsymbol{b} \in I_{S_{*}}} H_{(\boldsymbol{a}, \boldsymbol{b})} \boldsymbol{V}_{\boldsymbol{b}}=\sum_{\boldsymbol{b} \in I_{S_{*}}} F_{Q}^{-1} \Delta\left(H_{(\boldsymbol{a}, \boldsymbol{b})}\right) F_{Q}^{-1} \boldsymbol{V}_{\boldsymbol{b}}
$$




$$
=F_{Q}^{-1}\left(\sum_{\boldsymbol{b} \in I_{S_{*}}} \Delta\left(H_{(\boldsymbol{a}, \boldsymbol{b})}\right) F_{Q}^{-1} \boldsymbol{V}_{\boldsymbol{b}}\right) .
$$

Parallelism is readily seen in (26). We assume that $P$ processors are available. For simplicity, $P$ is assumed to be a power of two that divides $\Lambda$. We denote the ratio by $\rho=\Lambda / P$. We decompose $V$ in $P$ groups of size $\rho Q$ and denote the set of indices of the $i$-th group by $P_{i}$. Thus, processor $i$ receives the collection of input vector segments $V_{\boldsymbol{b}}$, with $\boldsymbol{b} \in P_{i}$. A precomputed array of size $\Lambda Q$ containing the diagonal entries of $\Delta\left(H_{(\boldsymbol{a}, \boldsymbol{b})}\right)$, is stored in processor $i$, as well. This array is denoted $D_{\boldsymbol{b}}$. For a $Q$-point vector $Y$, we define $D_{\boldsymbol{b}} \odot Y$ as the Hadamard product of the vector formed by concatenating $\Lambda$ copies of $Y$ and $D_{\boldsymbol{b}}$. Using these conventions, we translate formula (26) into the next parallel crystallographic FFT (PCFFT) method. The first steps are:

Step 1. For each $i=0$ to $P-1$, processor $i$ computes $Y_{\boldsymbol{a}}=F_{Q}^{-1} V_{\boldsymbol{a}}$, for each $\boldsymbol{a} \in P_{i}$.

Step 2. For each $i=0$ to $P-1$, processor $i$ computes $Z_{\boldsymbol{a}}=D_{\boldsymbol{a}} \odot Y_{\boldsymbol{a}}$, for each $\boldsymbol{a} \in P_{i}$.

Step 3. For each $i=0$ to $P-1$, processor $i$ computes $X_{i}=\sum_{\boldsymbol{a} \in P_{i}} Z_{\boldsymbol{a}}$.

Steps 1 to 3 require no interprocessor communication. However, in order to complete the sum in (26), all $\Lambda Q$-point data vectors $X_{i}, o \leq i \leq P-1$; must be added together. Interprocessor communications and vector additions overlap while computing this sum. Our communication topology is a hypercube. At each step, a different set consisting of all the parallel edges of the hypercube is selected. At each step, each pair of processors linked by by a hypercube edge, exchange data. There will be $\log P$ sets of parallel edges. Thus, for each $0 \leq j \leq \log P-1$, we denote by $E_{j}$ one such set of parallel hypercube edges.

Step 4. For each $j=0, \ldots, \log P-1$, for each pair $(i, l) \in E_{j}$, processor $i$ sends the upper segment of $X_{i}$ to processor $l$, and processor $l$ sends the lower segment of $X_{l}$, to processor $i$. Then, processor $i$ adds the lower segment of $X_{l}$ to the lower segment of $X_{i}$, and stores the result a new, half size vector $X_{i}$; while processor $l$ adds the upper segment of $X_{i}$ with the upper segment of $X_{l}$, and stores the result in a new, half size vector $X_{l}$.

At the end of step 4, processor $i$ holds in $X_{i}$, a $\rho Q$-point segment of the sum in (26). The core computation is completed by performing

Step 5. For each $i=0$ to $P-1$, processor $i$ computes $U_{\boldsymbol{a}}=F_{Q}^{-1} X_{\boldsymbol{a}}^{(i)}$, for each $a \in P_{i}$.

The addition of datum $f(\mathbf{0})$, which is part of the border computation, is performed as follows

Step 6. For each $i=0$ to $P-1$, processor $i$ computes $\hat{f}\left(O_{M}^{l_{\boldsymbol{a}}}(\boldsymbol{a})=\frac{1}{\sqrt{N}}[f(\mathbf{0})]_{l_{\boldsymbol{a}}}+\right.$ $\boldsymbol{U}_{\boldsymbol{a}}^{*}$, for each $\boldsymbol{a} \in P_{i}$. Here $\boldsymbol{U}_{\boldsymbol{a}}^{*}$ is the column vector formed by the $L_{\boldsymbol{a}}$ first entries of the vector $\boldsymbol{U}_{\boldsymbol{a}}$ computed in step 5 . 
Finally, the computation of $\hat{f}(\mathbf{0})$ is not worth parallelizing. We simply perform

Step 7. $\hat{f}(\mathbf{0})=\frac{1}{\sqrt{N}} \sum_{\boldsymbol{b} \in I_{S_{*}}} f(\boldsymbol{b})\left|O_{S}(\boldsymbol{b})\right|$. Here $\left|O_{S}(\boldsymbol{b})\right|$ is the size of $O_{S}(\boldsymbol{b})$.

The contribution to the time complexity of the PCFFT of steps 6 and 7 is negligible. Steps 1 and 5 are performed in $O(\rho Q \log Q)$ time. Steps 2 and 3 are performed in $O(\rho \Lambda Q)$ time. Step 4 is performed in $\log P$ passes. The total number of data-points transmitted in parallel is $\Lambda Q$, and a similar number of parallel additions are performed. Thus, this step is $O(\Lambda Q)$.

\section{Computer Experiments}

Following [5], we consider an $S$-symmetric input array whose redundancies are described by the symmetry

$$
S=\left[\begin{array}{rrr}
0 & 0 & 1 \\
0 & 1 & 0 \\
-1 & 0 & 0
\end{array}\right] \text {. }
$$

We choose three-dimensional data arrays of several prime edge-lengths, and compute the the corresponding parameters $Q$ and $\Lambda$. The results are shown in Table 1. We kept $Q=2 L-1$ in all our computer runs. We implemented our algorithm in C/MPI and run it on a commodity cluster conformed by eight 650 $\mathrm{MHz}$ Pentium III dual processors, interconnected by a 16 -port switch. The cluster runs under Linux/OSCAR. The one-dimensional, $Q$-point FFTs in steps 1 and 5 of the PCFFT are computed with the 1-D FFTW [4] package.

Table 2 compares the execution times in seconds of the 3-D FFTW [4] and our parallel crystallographic FFT. We also computed the speed up ratios between the 3-D FFTW and the PCFFT. In doing this we had adopted the classical speed up definition as the ratio between the execution times of the parallel method and the best serial algorithm, for the same problem size. The less classical but more common speed-up definition, which is the ratio between the execution times of the parallel method in $P$ processors and the parallel method in one processor, is meaningless in our case, since the PCFFT in one processor is on average, far less efficient than the 3-D FFTW. We also compared the PCFFT run times with those of the parallel MPI-FFTW [4. It turns out, however, that due to

Table 1. PCFFT parameters for different problem sizes

\begin{tabular}{|c|c|c|c|}
\hline Prime Edge & Problem Size & $\Lambda$ & $Q$ \\
\hline 199 & 7880599 & 200 & 19799 \\
223 & 11089576 & 224 & 24863 \\
239 & 13651919 & 240 & 28599 \\
263 & 18191447 & 264 & 34583 \\
271 & 19902511 & 272 & 36719 \\
311 & 30080231 & 312 & 48359 \\
\hline
\end{tabular}


Table 2. 3-D FFTW vs. PCFFT

\begin{tabular}{|c|c|c|c|}
\hline Problem Size & 3-D FFTW time & PCFFT time & Speed up \\
\hline 7880599 & 19.887 & 3.018 & 6.59 \\
11089576 & 52.428 & 3.572 & 14.68 \\
13651919 & 61.019 & 5.184 & 11.77 \\
18191447 & 254.969 & 10.336 & 24.67 \\
19902511 & 269.612 & 5.803 & 46.46 \\
30080231 & 619.351 & 77.259 & 8.02 \\
\hline
\end{tabular}

Table 3. Performance of the Hypercube Reduce-Scatter vs. MPI Reduce-Scatter routine for computing step 4. Times are measured in seconds

\begin{tabular}{|c|c|c|}
\hline Problem Size & Hypercube R-S & MPI R-S \\
\hline 7880599 & .430 & .972 \\
11089576 & .563 & 1.121 \\
13651919 & .67 & 1.404 \\
18191447 & 1.142 & 1.735 \\
19902511 & .836 & 1.837 \\
30080231 & 45.496 & 54.512 \\
\hline
\end{tabular}

the load unbalancing induced by the irregularity of the problem, and above all, the large data array transpositions and communications that were required, the MPI-FFTW performed very poorly in our system. In fact, in most runs, the MPIFFTW took longer than the 3-D FFTW in our cluster. We could not compare the PCFFT with a parallel symmetrized FFT since, to our knowledge, no other parallel prime edge-length crystallographic FFT is currently available.

It is worth remarking that in the usual, asymmetric multidimensional FFT no overlaps between communications and computations are possible. This overlapping is, thus, an additional attractive computational feature of the PCFFT. As a way of assessing the impact of a careful exploitation of this feature in the overall PCFFT performance, we compare the performance of two implementations of step 4 of the PCFFT. The first uses an MPI Reduce-Scatter function, while the second, a communication/addition method tailor made for the problem. Results are reported in Table 3 . The tailor made routine, which we call hypercube method, has been crafted to maximize overlaps between communications and computations. The authors are still researching on further improvements on this step of the PCFFT which is crucial for the scaling of the method to massive distributed systems

Acknowledgments. This work was partially supported by NIH/NIGMS grant No. S06GM08103 and the NSF PRECISE project of the University of Puerto Rico at Mayagüez 


\section{References}

1. L. Auslander, and M. Shenefelt, Fourier Transforms that Respect Crystallographic Symmetries, IBM J. Res. and Dev., 31, pp. 213-223, 1987.

2. M. An, J.W. Cooley, and R. Tolimeri, Factorization Methods for Crystallographic Fourier Transforms, Advances in Appl. Math. 11, pp. 358-371, 1990.

3. J. Cooley, and J. Tukey, An Algorithm for the Machine Calculation of Complex Fourier Series, Math. Comp., 19, pp. 297-301, 1965.

4. M. Frigo, S.G. Johnson, An adaptive software architecture for the FFT ICASSP Conference Proceedings, 3 (1998), pp 1381-1384.

5. J. Seguel, D. Bollman, E. Orozco, A New Prime Edge-length Crystallographic FFT, ELSEVIER Lecture Notes in Computer Science, Vol. 2330, pp. 548-557,2002.

6. J. Seguel, A Unified Treatment of Compact Symmetric FFT Code Generation, IEEE Transactions on Signal Processing, Vol 50, No. 11, pp. 2789-2797, 2002.

7. L.F. Ten Eyck, Crystallographic Fast Fourier Transforms, ACTA Crystallogr. A, vol. 29, pp. 183-191, 1973.

8. C.M. Weeks, G.T. DeTitta, H.A. Hauptman, H.A Thuman, R. Miller, Structure Solution by Minimal Function Phase Refinement and Fourier Filtering: II Implementation and Applications, Acta Crystallogr. A50, pp. 210-220, 1994.

9. C.M. Weeks, R. Miller Optimizing Shake-and-Bake for Proteins, Acta Crystallogr. D55, pp 492-500, 1999. 